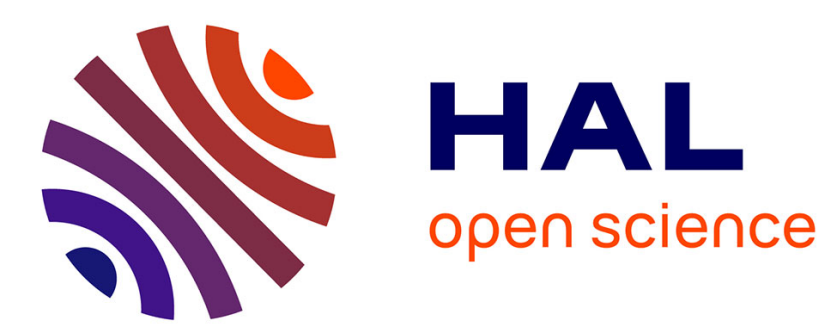

\title{
Study and choice of actuation for a walking assist device
}

Christine Chevallereau, Yannick Aoustin, Vigen Arakalian

\section{To cite this version:}

Christine Chevallereau, Yannick Aoustin, Vigen Arakalian. Study and choice of actuation for a walking assist device. Mechanism and Machine Science, New trends in medical and service robots, Springer, 2015., 2015. hal-01178930

\section{HAL Id: hal-01178930 \\ https://hal.science/hal-01178930}

Submitted on 21 Jul 2015

HAL is a multi-disciplinary open access archive for the deposit and dissemination of scientific research documents, whether they are published or not. The documents may come from teaching and research institutions in France or abroad, or from public or private research centers.
L'archive ouverte pluridisciplinaire HAL, est destinée au dépôt et à la diffusion de documents scientifiques de niveau recherche, publiés ou non, émanant des établissements d'enseignement et de recherche français ou étrangers, des laboratoires publics ou privés. 


\title{
Study and choice of actuation for a walking assist device
}

\author{
Y. Aoustin, C. Chevallereau, and V. Arakalian \\ L'UNAM, Institut de Recherche en Communications \\ et Cybernétique de Nantes, UMR 6597, \\ CNRS, École Centrale de Nantes, Université de Nantes. \\ 1, rue de la Nö̈, BP 92101. 44321 Nantes, France. \\ e-mail: Yannick. Aoustin@irccyn. ec-nantes. fr, \\ e-mail: Christine. Chevallereau@irccyn. ec-nantes. fr, \\ e-mail: Vigen. Arakalian@irccyn. ec-nantes. fr
}

\begin{abstract}
A walking assist device (WAD) with bodyweight support reduces energy expenditure of a walking person. However, it is also important that the location of actuators in the WAD will be optimally chosen. For this purpose a wearable assist device composed of a bodyweight support, legs and shoes articulated with hip (upper joint), knee (middle joint), and ankle (lower joint) is discussed. Since human walk involves large displacements only in sagittal plane, a planar model is considered. In order to evaluate the optimal distribution of input torques, a bipedal model of a seven-link system with several walking velocities is coupled with the mentioned WAD. To study the efficiency of the WAD and to choose an appropriate actuation, the torque cost is evaluated when the same walking pattern are tracked with and without a WAD. The paper deals with the torque cost for the human and the WAD with several types of actuation. It is shown that full actuation with six motors or partial actuation with two motors located at the upper joints are two more efficient solutions while an actuation at the middle joints or lower joints only is ineffective. The numerical simulations carried out for several walking velocities confirm the mentioned observations.
\end{abstract}

Key words: assist device, walking gait, optimization, torques cost, biped fully assisted, biped partially assisted.

\section{Introduction}

Musculoskeletal disorders for workers and/or elderly people are actually social worries. As a consequence during the last few years, several biomechanic studies and realizations of walking assist devices have been carried out. For example, Priebe and Kram [1] compared the metabolic power consumption for ten young adults walking without assistance and using two-wheeled, fourwheeled and four-footed walker devices. Zhang and Hashimoto [2] presented a trajectory generation method for a robotic suit to assist walking by supporting the hip joints. Ikeuchi et al [3] proposed a wearable walking assist device. This device, with a seat, segments, and two shoes is disposed along 
the inner side of the user's legs. It produces an assisting force directed from the center of pressure to the center of mass of the human using two actuators.

The design of a WAD remains an open problem since walking implies displacement of the mass and inertia of the WAD, the human walking must be facilitated and not perturbed. Human's walking is a complex coordination of muscle activity, actuator torques and joint motions. This is even more true for human's walking with an assist device, where closed kinematic chains has to be considered. This paper deals with the problem of the best distribution of actuators for a given wearable walking device which assists a seven-link planar biped during a given cyclic walking gait.

To evaluate the effect of WAD on the torque cost, reference walking gaits must previously be defined. For example the cart-model, or the linear inverted pendulum model, based on the zero moment point $Z M P$ can be efficient to design a walking gait; see [4], [5], and [6]. A walking gait based on capture point regulation is developed in [7] and in [8]. The capture point is the location on the ground where a biped needs to step in order to come to a stop. These approaches are largely used in humanoid robotics but do not produce humanlike motion and thus are not well adapted in our context. Another approach employed to generate walking patterns for biped robots is based on central pattern generators (CPGs) and do not require any physical model of the biped; see [9] and [10]. The reference motion of the biped can be based on record of human motion [11], [12]. In this paper walking patterns defined through an optimization algorithm.

To study the efficiency of the walking assist devices, the torques of the model of human without and with the wearable device will be compared. Several levels of assistance will be considered. These levels of assistance are respectively the biped fully assisted, the biped partially assisted through two motors located at the upper joints, at the middle joints or at the lower joints respectively. The structure of the paper is the following. In section 2 , the modeling of the biped and its walking assist device are defined. In section 3, the design of reference gaits is shortly presented. Section 4 is devoted to the statement of the considered problem. In section 5, the numerical tests are discussed. Section 6 offers our conclusion and perspectives.

\section{Studied biped with its wearable walking assist device}

The wearable walking assist device, drawn with thick lines in Figure 1, is composed of a seat, attached to the waist, segments, and two shoes. It has six joints in the sagittal plane, i.e. two upper joints between the seat and the upper legs, two middle joints between the upper legs and the lower legs, and two lower joints between the lower legs and the feet. The wearable device has a similar shape than of human with two shins, femurs and shoes. The connection of the wearable walking assist device with human corresponds to 
the complete feet and to the base of the trunk according to Figure 1. The parameters of the human model is taken from the literature [13]. The whole mass of the biped is $75 \mathrm{~kg}$, its height is $1.75 \mathrm{~m}$. Table 1 summarizes the parameters of the model of human and the WAD. The arms and head of the human are taken into account in the parameters of the trunk.

\begin{tabular}{|c|c|c|c|c|}
\hline & Mass $(\mathrm{kg})$ & Length $(\mathrm{m})$ & Inertia $\left(\mathrm{kg} \cdot \mathrm{m}^{2}\right)$ & center of mass $(\mathrm{m})$ \\
\hline $\begin{array}{c}\text { Foot } \\
\text { and shoe }\end{array}$ & $m_{f}=0.678$ & $\begin{array}{c}L_{p}=0.207 \\
l_{p}=0.072 \\
H_{p}=0.064\end{array}$ & $I^{f}=0.012$ & $s_{p x}=0.0135$ \\
& & & $s_{p y}=0.0321$ \\
\hline Shin & $m_{s}=4.6$ & $l_{s}=0.497$ & $I^{s}=0.0521$ & $s_{s}=0.324$ \\
\hline Thigh & $m_{t}=8.6$ & $l_{t}=0.41$ & $I^{t}=0.7414$ & $s_{t}=0.18$ \\
\hline Trunk & $m_{T}=16.5$ & $l_{T}=0.625$ & $I^{T}=11.3$ & $s_{T}=0.386$ \\
\hline Seat & $m_{3}=2.0$ & $l_{3}=0.1$ & $I^{T}=0.3$ & $s_{3}=0.05$ \\
\hline Upper link & $m_{1}=3.0$ & $l_{1}=0.392$ & $I^{1}=0.04$ & $s_{1}=0.1127$ \\
\hline Lower link & $m_{2}=2.0$ & $l_{2}=0.3645$ & $I^{2}=0.02$ & $s_{2}=0.169$ \\
\hline
\end{tabular}

Table 1 Parameters for the seven-link biped and the walking assist device.

The model of the human equipped with the walking assist device includes mechanical closed loops due to the multiple connections between the human and the WAD. The generalized configuration vector $\mathbf{x}$ is

$$
\mathbf{x}=\left[q_{1}, q_{2}, q_{3}, q_{4}, q_{5}, q_{6}, q_{7}, q_{8}, q_{91}, q_{92}, q_{101}, q_{102}, q_{11}, x, y\right]^{\top} .
$$

where the configuration variables are described in figure 1.

The dynamic model is calculated using the equivalent tree structure and adding the external forces and moments between the cut links as external forces and moments [14]. Let us define by $\mathbf{f}_{c_{i}}=\left[f_{x_{i}}, f_{y_{i}}, m_{z_{i}}\right]^{\top}, i=1,2$, the vectors composed of the external forces and moments for both loop closures denote action torsors. Through the virtual work principle, these vectors contribute into the dynamic model by adding terms $\mathbf{J}_{i}^{\top} \lambda_{i}$, where $\mathbf{J}_{i}$ is the $3 \times 15$ Jacobian matrix describing how the action torsor acts on the system.

The model of human and its WAD is also subject of external forces and moments exerted by the ground in the stance feet. The complete dynamic model can be written as:

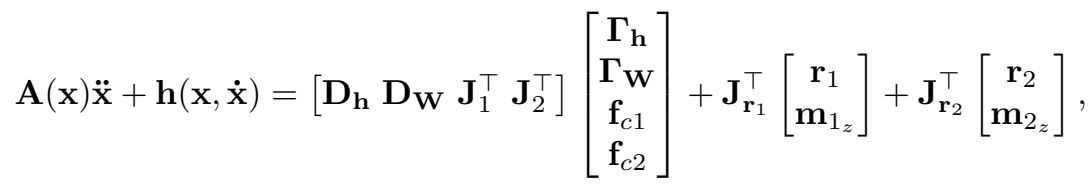

with the constraint equations,

$$
\begin{gathered}
\mathbf{J}_{\mathbf{r}_{i}} \ddot{\mathbf{x}}+\dot{\mathbf{J}}_{\mathbf{r}_{i} \dot{\mathbf{x}}}=\mathbf{0} \text { for } i=1 \text { to } 2, \\
{\left[\begin{array}{c}
\mathbf{J}_{1} \\
\mathbf{J}_{2}
\end{array}\right] \ddot{\mathbf{x}}+\left[\begin{array}{l}
\dot{\mathbf{J}}_{1} \\
\mathbf{J}_{2}
\end{array}\right] \dot{\mathbf{x}}=\mathbf{0} .}
\end{gathered}
$$



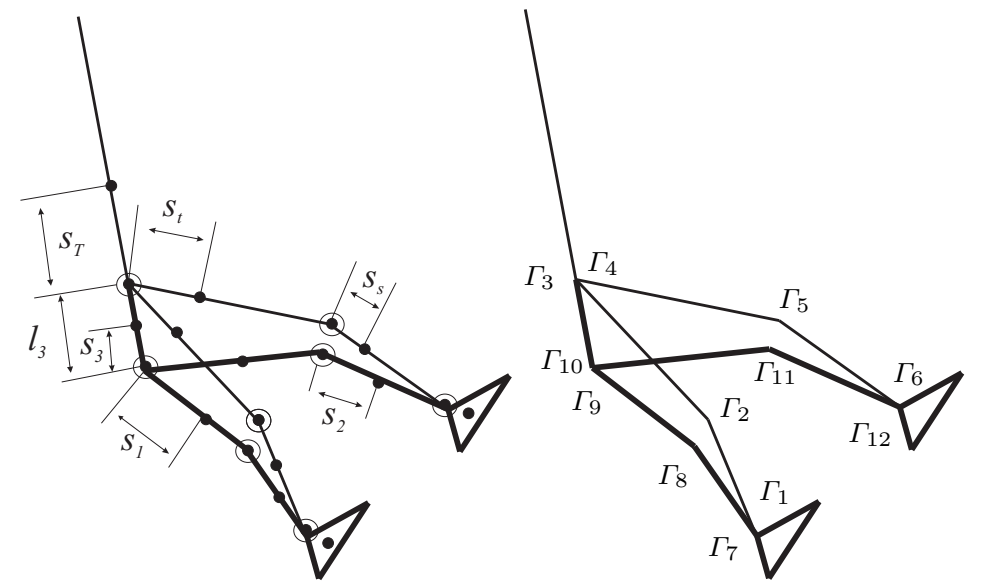

(a)

(b)

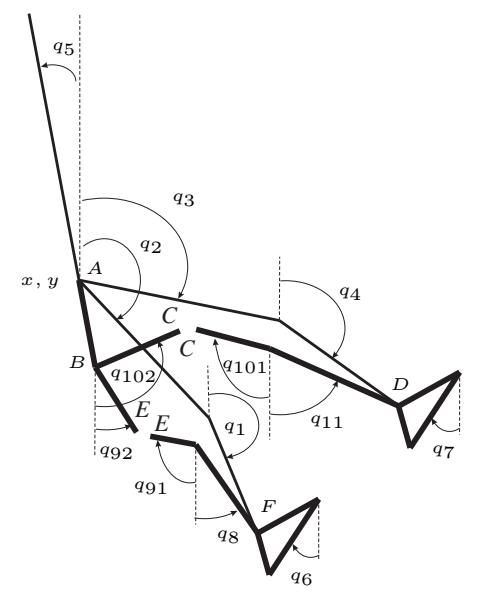

(c)

Fig. 1 Planar model of human with its walking assist device. The model of human is depicted in fine line, the coordinates, which are used to describe the human configuration include the position and orientation of the trunk defined by $x, y, q_{5}$ and the orientation of the thigh, shin and feet defined by $q_{1}, q_{2}, q_{3}, q_{4}, q_{6}, q_{7}$. The WAD shown in thick line is attached to the waist of the human, and the articulation of the seat is below the human hip with a distance $l_{3}$ along the trunk. The WAD is also attached to the human throughout the feet. Mechanical loops are thus created and the modeling required to cut these closed loops virtually 1 (c) and to introduce the coordinates $q_{8}, q_{91}, q_{92}, q_{101}, q_{102}, q_{11}$ to described the WAD configuration.

$\left[\mathbf{r}_{i} \mathbf{m}_{i_{z}}\right]^{\top}$, with $i=1$ to 2 , are the resultant action torsors of the contact efforts with the ground reaction in both feet. $\mathbf{J}_{r_{1}}$ and $\mathbf{J}_{r_{2}}$ are the $3 \times 15$ Jacobian matrices for the constraint equations in position and orientation for both feet, respectively. $\mathbf{A}(\mathbf{x})$ is the $15 \times 15$ symmetric positive definite inertia 
matrix, $\mathbf{h}(\mathbf{x}, \dot{\mathbf{x}})$ is the $(15 \times 1)$ vector, which groups the centrifugal, Coriolis effects, and the gravity forces. The $(15 \times 6)$ constant matrix $\mathbf{D}_{\mathbf{h}}$, describes the action of the torque $\boldsymbol{\Gamma}_{\mathbf{h}}$ of the human model on the system. The $\left(15 \times n_{a}\right)$ constant matrix $\mathbf{D}_{\mathbf{W}}$, describes how the torque $\boldsymbol{\Gamma}_{W}$ of the WAD act on the system, here $n_{a}$ is the number of actuators $2 \leq n_{a} \leq 6$. In the single support, with a stance foot and with flat foot contact on the ground the number of degrees of freedom is six, since the constraints (3) have to be satisfied.

\section{The reference cyclic walking gait}

The goal of this study is to compare the energy consumption for a given reference walking gait adopted by the biped alternately without and with its wearable assist device.

Initially, the reference walking gait is computed for the biped without the WAD. This walking gait is cyclic, i.e. all steps are identical. Each step is composed of a single support phase and an impact. In single support the stance foot has a flat contact with the ground. When the swing foot impacts the ground the other foot takes off. The evolution of each joint of the biped is prescribed with a third order polynomial function, of which the coefficients are calculated through a parametric optimization algorithm. The criterion for minimization is the integral of the square of torques for a given walking step length at several velocities; see [15] and [16]. The cyclic walking gait is defined such that the conditions of the flat contact for the stance foot are satisfied without sliding nor rotation of this stance foot. This gait has been chosen for its simplicity, more complex gait with double support phase can be considered in future.

\section{Study of the optimal distribution of the torques for the biped with the wearable assist device}

The biped equipped of a WAD tracks the walking cyclic gait defined in Sec. 3 . The optimal distribution of the torques for the biped and its walking assist device is studied for the four following cases. Firstly the biped is fully assisted, the WAD provides six torques. The three other cases are defined such as the WAD provides two torques $\left(\Gamma_{7}\right.$ and $\left.\Gamma_{8}\right),\left(\Gamma_{9}\right.$ and $\left.\Gamma_{10}\right)$, or $\left(\Gamma_{11}\right.$ and $\left.\Gamma_{12}\right)$. For each case, at each sampling time during one step of the cyclic gait, an optimization algorithm is stated to define the torque distribution between human and WAD.

Let us multiply the dynamic model (2) with the matrix $\mathbf{J}^{\perp}(12 \times 15)=$ $\left[\begin{array}{ll}\mathbf{J}_{\mathbf{r}_{1}}^{\top} & \mathbf{J}_{\mathbf{r}_{2}}^{\top}\end{array}\right]^{\perp}$ to obtain a model, which does not depend on the ground reaction on the stance foot: 


$$
\mathbf{J}^{\perp} \mathbf{A}(\mathbf{x}) \ddot{\mathbf{x}}+\mathbf{J}^{\perp} \mathbf{h}(\mathbf{x}, \dot{\mathbf{x}})=\mathbf{J}^{\perp}\left[\begin{array}{lll}
\mathbf{D} & \mathbf{J}_{1}^{\top} & \mathbf{J}_{2}^{\top}
\end{array}\right]\left[\begin{array}{c}
\boldsymbol{\Gamma}_{\mathbf{h}} \\
\boldsymbol{\Gamma}_{\mathbf{W}} \\
\mathbf{f}_{c 1} \\
\mathbf{f}_{c 2}
\end{array}\right] .
$$

The left hand side of (4) depends only of the reference walking gait, and thus is known. The objective is now to find the forces and moments $\mathbf{f}_{c 1}, \mathbf{f}_{c 2}$ and the torques $\boldsymbol{\Gamma}_{\mathbf{h}}, \boldsymbol{\Gamma}_{\mathbf{W}}$ that produce the desired motion. It exists an infinite number of solutions since equation (4) is a system of 12 equations and $12+$ $n_{a}$ unknowns, with a full rank matrix $\left[\mathbf{D} \mathbf{J}_{1}^{\top} \mathbf{J}_{2}^{\top}\right]$. The dimension of overactuation is $n_{a}$, the number of actuators of the WAD.

Two criteria will be considered depending on the objective of the use of the WAD. For people with disabilities, the minimisation of the human action is an obvious criterion

$$
C_{1}=\boldsymbol{\Gamma}_{\mathbf{h}}^{\top} \boldsymbol{\Gamma}_{\mathbf{h}}
$$

For valid people, the minimisation of the human and WAD torques can also be used:

$$
C_{2}=\boldsymbol{\Gamma}_{\mathbf{h}}^{\top} \boldsymbol{\Gamma}_{\mathbf{h}}+\boldsymbol{\Gamma}_{\mathbf{W}}^{\top} \boldsymbol{\Gamma}_{\mathbf{W}} .
$$

The weighted combination of the two criteria can be considered, but as illustration the two extreme cases $C_{1}$ and $C_{2}$ will only be studied. Constraints are defined to limit the efforts of each loop closure between the human and its assist advice, to ensure that the vertical component of the ground reaction on the stance foot is positive. Furthermore a constraint of no rotation of the stance foot is also taken into account.

\section{Results}

The results are presented for a walking speed $0.65 \mathrm{~m} / \mathrm{s}(2.34 \mathrm{~km} / \mathrm{h})$ of the biped, considering respectively the optimized criterion $C_{1}$ and the optimized criterion $C_{2}$ given in figures 2 and 3 .

Four different cases of actuation of the WAD are considered: the WAD is fully actuated, or partially actuated through the actuated upper joints only $\left(\Gamma_{9}=0, \Gamma_{10}=0, \Gamma_{11}=0\right.$ and $\left.\Gamma_{12}=0\right)$, the actuated middle joints only $\left(\Gamma_{7}=0, \Gamma_{8}=0, \Gamma_{11}=0\right.$ and $\left.\Gamma_{12}=0\right)$, and the upper joints only $\left(\Gamma_{7}=0\right.$, $\Gamma_{8}=0, \Gamma_{9}=0$ and $\left.\Gamma_{10}=0\right)$. The results are compared to the case of the walking without assistance. In this case the biped is not equipped with the $\mathrm{WAD}$, thus the weight of the walking assist device is not taken into account. These results correspond to the nominal case for the definition of the cyclic walking (see section 3). The shown results correspond to the following cases.

1. The torque cost for the model of human expressed as $\frac{1}{d} \int_{0}^{T} \Gamma_{h}^{\top} \Gamma_{h} \mathrm{~d} t$, where $d$ is the walking step length when the human is equipped with the WAD, 
2. The torque cost for the WAD expressed as $\frac{1}{d} \int_{0}^{T} \Gamma_{W}^{\top} \Gamma_{W} \mathrm{~d} t$,

3. The sum of these two quantities,

4. The torque cost for the model of human expressed as $\frac{1}{d} \int_{0}^{T} \Gamma_{h}^{\top} \Gamma_{h} \mathrm{~d} t$, when the human is not equipped with the WAD.

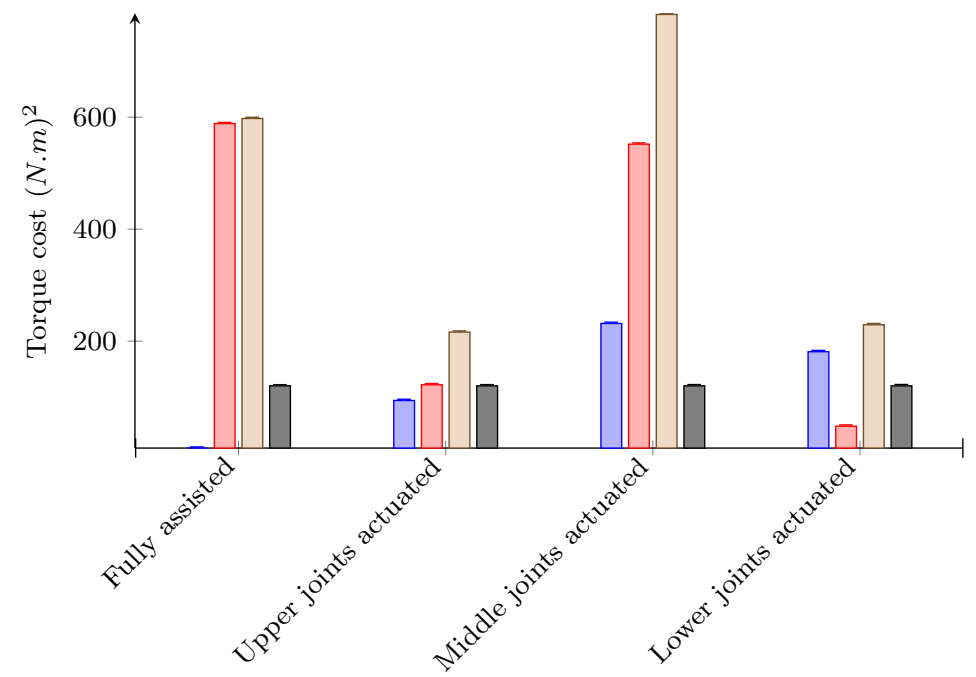

पu Part for Assisted Biped Qu Part for Assist device प■Biped with Assist device D० Unassisted Biped

Fig. 2 Histogram of torque cost for the different distributions of the actuation of the WAD, and minimization of criterion $C_{1}$ (Only the human torques are minimized)

Based on the obtained results, we following observations can be made

1. An actuation of the middle joints or of the lower joints only are not appropriate since the torque cost for the human increases with these types of assistance.

2. A complete actuation of the WAD, allows a displacement of the human without any human torque if necessary (see figure 2). In this case, the cost to be produced by the WAD is high. A decrease of the human torque cost with respect to an unassisted case can be obtained with a very low increase of the total torque cost when criterion $C_{2}$ is minimized. The human torque cost can be adjusted between 0 and this value through the use of a weighted criterion $C_{1}+\alpha C_{2}$.

3. The solution of the assistance in the upper joints only seems efficient to help the biped in good health, the device at least compensates for its weight, it can also reduce the burden of the human according to the optimized criterion. 


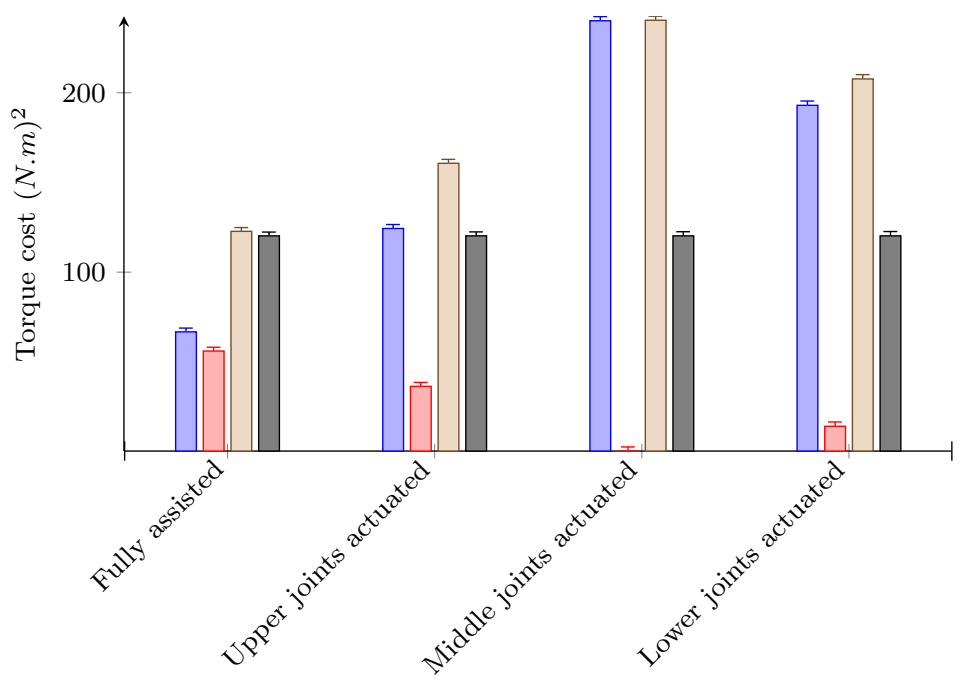

Tu Part for Assisted Biped प Part for Assist device प $\mathrm{B}$ Biped with Assist device Lu Unassisted Biped

Fig. 3 Histogram of torque cost for the different distributions of the actuation of the WAD, and minimization of criterion $C_{2}$ (The torques of the biped and walking assist device are minimized).

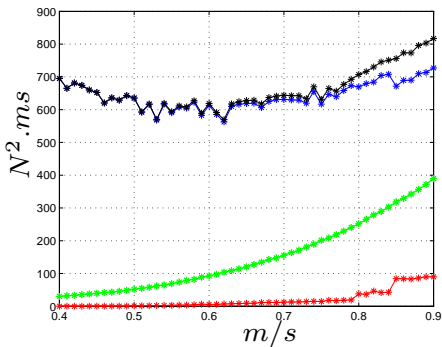

(a)

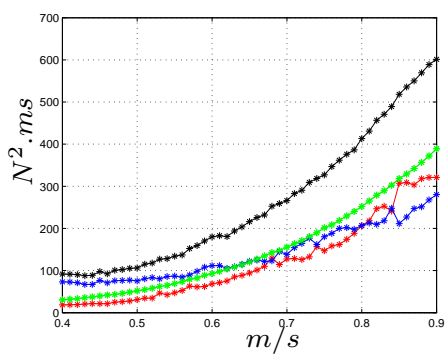

(c)

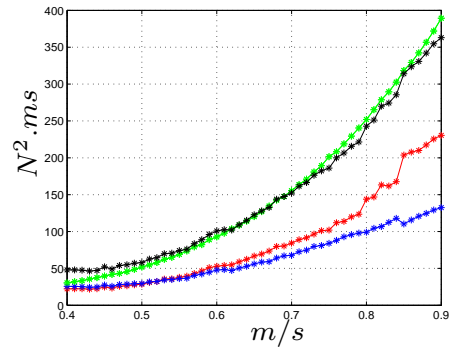

(b)

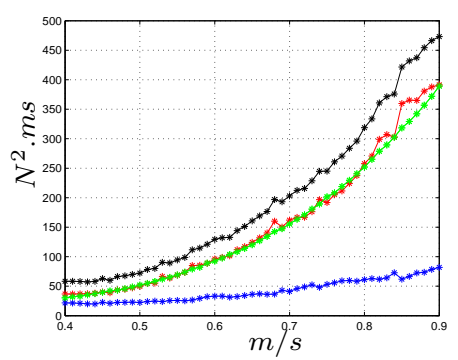

(d)

Fig. 4 Torque cost as function of the motion velocities: Biped without assistance (green), Part for the WAD (blue), Part for the human model (red), Addition of the WAD and model of human parts (black). (a) Fully actuated WAD, optimized criterion $C_{1}$; (b) Fully actuated WAD, optimized criterion $C_{2}$; (c) Upper joint actuation of the WAD only, optimized criterion $C_{1}$; (d) Upper joint actuation of the WAD only, optimized criterion $C_{2}$ 
Figures 4(a)-4(d) present the torque cost previously studied, as function of the motion velocity $V$ of the biped with and without its WAD. The velocities vary between $0.4 \mathrm{~m} / \mathrm{s}$ and $0.9 \mathrm{~m} / \mathrm{s}(1.44 \mathrm{~km} / \mathrm{h}$ and $3.24 \mathrm{~km} / \mathrm{h})$. Figures 4 (a) and 4 (b) are relative to the fully assisted biped for the optimized criteria $C_{1}$ and $C_{2}$ respectively. The cost torque for the model of human of the fully assisted biped is smaller than without assistance. When $C_{1 k}$ is the optimization criterion, the torque cost of the WAD is high. However the torques of the model of human are not equal to zero and even increase when the velocity is over $0.6 \mathrm{~m} / \mathrm{s}(2.88 \mathrm{~km} / \mathrm{h})$. This is due to the fact that the reference trajectory is adapted to the autonomous biped and not to the biped with its WAD. In case of a paralyzed human using a walking assist device, the gait has to be adapted to the complete system. Figures 4(c) and 4(d) are relative to the model of human equipped of the WAD actuated at the upper joint level only, for the optimized criterion $C_{1}$ and $C_{2}$ respectively. Depending of the criterion minimized, the torque cost for the human can be reduced or almost unchanged with respect to the case of the human not equipped with a WAD. These curves confirm that to increase the autonomy of the biped the assistance in the upper joints only is a good compromise.

\section{Conclusion}

The paper presented a comparative analysis of the torque cost of walking with several actuations of a WAD in the case of simple walking gait with single support phases and impacts. It has been shown that the WAD fully actuated and the WAD actuated only at the upper joints are two interesting proposals. The WAD only actuated at the middle joints or at the lower joints are ineffective. A study for several walking velocities confirmed these results. It is an initial step to start the process of conception of a walking assist device. The perspective is a deeper study of the optimal placement of the actuators by introducing more complex and anthropomorphic walking gaits and the extension to the introduction of passive element to store energy.

Acknowledgements This work is supported by Ministry of Education and Science of Russian Federation and by Région des Pays de la Loire, Project LMA and Gérontopôle Autonomie Longévité des Pays de la Loire. 


\section{References}

1. J. R. Priebe and R. Kram, "Why is walker-assisted gait metabolically expensive?" Gait \& Posture, vol. 34, pp. 265-269, 2011.

2. X. Zhang and M. Hashimoto, "Synchronization-based trajectory generation method for a robotic suit using neural oscillators for hip joint support in walking," Mechatronics, vol. 22, pp. 33-44, 2012.

3. Y. Ikeuchi, J. Ashihara, Y. Hiki, H. Kudoh, and T. Noda, "Walking assist device with bodyweight support system," in Proc. IEEE/RSJ Int. Conf on Intelligent Robots and Systems, St Louis, USA, 2010, pp. 4073-4079.

4. M. Vukobratovic and B. Borovac, "Zero-moment point-thirty five years of its life," Int. J. of Humanoid Robotics, vol. 1, no. 1, pp. 157-173, 2004.

5. K. Kaneko, F. Kanehiro, S. Kajita, H. Hirukawa, T. Kawasaki, M. Hirata, K.Akachi, and T. Isozumi, "Humanoid robot hrp-2," in Proc. IEEE Int. Conf. on Robotics and Automation ICRA, New-Orleans, Louisiana, USA, 2004, pp. 1083-1090.

6. A. D. Kuo, J. M. Donelan, and A. Ruina, "Energetic consequences of walking like an inverted pendulum: Step-to-step transitions," Exercise and Sport Sciences Reviews, vol. 33, no. 2, pp. 88-97, 2005.

7. J. Englsberger, C. Ott, M. A. Roa, A. Albu-Shäffer, and G. Hirzinger, "Bipedal walking control based on capture point dynamics," in Proc. Int. Conf. on Intelligent Robots and Systems (IROS), San Francsico, USA, 2001, pp. 4420-4427.

8. J. E. Pratt, T. Koolen, T. de. Boer, J. R. Rebula, S. Cotton, J. Carff, M. Johnson, and P. D. Neuhaus, "Capturability-based analysis and control of legged locomotion, part 2: application to $\mathrm{m} 2 \mathrm{v} 2$, a lower-body humanoid," The International Journal of Robotic Research, vol. 31, no. 10, pp. 1117-1133, 2012.

9. S. Behnke, "Online trajectory generation for omnidirectional biped walking," in Proc. Int. Conf. on Robotics and Automation (ICRA), Orlando, USA, 2006, pp. 1597-1603.

10. C. Graf, A. Häryl, T. Röfer, and T. Laue, "A robust closed-loop gait for the standard platform league humanoid," in Proc. Workshop on Humanoid Soccer Robots of the IEEE-RAS Int. Conf. on Humanoid Robots, Paris, France, 2009, pp. 30-37.

11. M. J. Powell, A. Heraeid, and A. D. Ames, "Speed regulate in 3d robotic walking through motion transitions between human-inspired partial hybrid zero dynamics," in Proc. IEEE Int. Conf. on Robotics and Automation (ICRA), Karlsruhe, Germany, 2013, pp. 4803-4810.

12. M. Tomic, C. Vassallo, C. Chevallereau, A. Rodic, and V. Potkonjak, "Arms motion of a humanoid inspired by human motion," in Proc. Int. Conf. Medical and Service Robotics (MESROB 2014), Lausanne, Switzerland, 2014.

13. A. M. Formalskii, Locomotion of Anthropomorphic Mechanisms. [In Russian], Nauka, Moscow, Russia, 1982.

14. W. Khalil and E. Dombre, Modeling, identification and control of robots. Butterworth Heinemann, 2002.

15. V. V. Beletskii and P. S. Chudinov, "Parametric optimization in the problem of bipedal locomotion," Izv. An SSSR. Mekhanika Tverdogo Tela [Mechanics of Solids], no. 1, pp. 25-35, 1977.

16. C. Chevallereau and Y. Aoustin, "Optimal reference trajectories for walking and running of a biped," Robotica, vol. 19, no. 5, pp. 557-569, 2001. 